\title{
PEGylated PLGA-based phase shift nanodroplets combined with focused ultrasound for blood brain barrier opening in rats
}

\author{
Xiang Zhang ${ }^{1,2}$, Jiangang Hu ${ }^{1,2}$, Guanjian Zhao ${ }^{1,2}$, Ning Huang ${ }^{1}$, Ying Tan $^{1}$, Li $\mathrm{Pi}^{1}$, \\ Qing Huang ${ }^{1}$, Feng Wang ${ }^{1}$, Zhigang Wang ${ }^{2}$, Zhibiao Wang ${ }^{3}$, Yuan Cheng ${ }^{1}$ \\ ${ }^{1}$ Department of Neurosurgery, The Second Affiliated Hospital of Chongqing Medical University, Chongqing, 400010, China \\ ${ }^{2}$ Chongqing Key Laboratory of Ultrasound Molecular Imaging, Institute of Ultrasound Imaging of Chongqing Medical \\ University, Chongqing, 400016, China \\ ${ }^{3}$ Chongqing Key Laboratory of Biomedical Engineering, College of Biomedical Engineering of Chongqing Medical University, \\ Chongqing, 400016, China
}

Correspondence to: Yuan Cheng, email: chengyuan023@aliyun.com

Keywords: blood brain barrier, focused ultrasound, acoustic droplet vaporization, perfluoropentane, PLGA

Received: January 17, $2017 \quad$ Accepted: April 05, $2017 \quad$ Published: April 17, 2017

Copyright: Xiang Zhang et al. This is an open-access article distributed under the terms of the Creative Commons Attribution License 3.0 (CC BY 3.0), which permits unrestricted use, distribution, and reproduction in any medium, provided the original author and source are credited.

\section{ABSTRACT}

Previous studies have shown that focused ultrasound (FUS) combined with systematic administration of microbubbles (MBs) can open the blood brain barrier (BBB) locally, transiently and reversibly. However, because of the micro size diameters, MBs are restricted in the intravascular space and cannot extravasate into diseased sites through the opened BBB. In this study, we fabricated one kind of nanoscale droplets which consisted of encapsulated liquid perfluoropentane cores and poly (ethyleneglycol) - poly (lactide-co-glycolic acid) shells. The nanodroplets had the capacity to realize liquid to gas phase shift under FUS. Significant extravasation of Evan's blue appeared when acoustic pressure reached 1.0 MPa. Intracerebral hemorrhages and erythrocyte extravasations were observed when the pressure was increased to 1.5 MPa. Prolonged sonication duration could enhance the level of BBB opening and broaden the time window simultaneously. Furthermore, compared with MBs, the distribution of EB extravasation was firmly confined within narrow region in the center of focal zone, suggesting the site of FUS induced BBB opening could be controlled with high precision by this procedure. Our results show the feasibility of serving PEGylated PLGA-based phase shift nanodroplet as an effective alternative mediating agent for FUS induced BBB opening.

\section{INTRODUCTION}

Blood-brain barrier (BBB) is the foremost obstacle which highly prevents effective therapeutic agents from transferring into brain parenchyma and functioning properly. For achieving an effective therapeutic concentration at diseased tissues, the doses of drugs that cannot cross BBB must be high enough. However, high drug doses can easily induce severe side-effects and undesired drug accumulation at non-diseased sites. Therefore, studies aimed at providing helpful ways for drug delivery across BBB with high efficiency are necessary. It has been demonstrated that focused ultrasound (FUS) could locally, transiently and reversibly increase the permeability of $\mathrm{BBB}$ in the presence of microbubbles(MBs), which shows tremendous potentials for targeted delivery of chemotherapeutic agents [1-3], antibodies [4, 5], genes [6,7], cells [8] and so on.

Conventional commercial or self-made MBs consist of inert gaseous cores and monolayer lipid shells which prevent fusion of bubbles and dissolution of gas into the surrounding medium. Under proper ultrasound pressure, MBs oscillate regularly and the repetitive contraction and expansion of MBs create microstreamings in nearby medium. This is known as stable cavitation [3,9]. The microstreamings could apply shear forces to the cerebrovascular endothelial cells and increase the cell permeability. When the pressure exceeds a certain level, MBs will collapse and generate forceful shock waves and microjets (called inertial cavitation). These powerful stresses can create pores within the cell membrane, break tight junctions between the adjacent brain endothelial 
cells, and finally enhance the transcellular and paracellular permeability of BBB [10]. Although these hypotheses are supported by convincing experimental data, the exact mechanism of FUS induced BBB opening in the presence of MBs is still unclear.

Because of the micron size diameters, the remaining MBs in circulation after sonication are too bulky to extravasate into brain parenchyma through the opened pathway. Additionally, MBs only have a circulation halflife of seconds to minutes [11] that re-administration during procedure is generally needed. Therefore, a new kind of mediating agent with smaller size and longer circulation half-life for FUS induced BBB opening is necessary.

Phase shift perfluorocarbon droplet, which generally consists of a liquid perfluorocarbon (PFC) core and a coating shell, can be excited by ultrasound and vaporize into gas bubbles if the acoustic power is high enough. This phenomenon is called acoustic droplet vaporization (ADV) [12-15]. The threshold for vaporization is increased with decreasing diameter and decreased with increasing ultrasound frequency and sonication time $[13,16]$. A predominant factor for successful vaporization of PFC droplets is the acoustic pressure [17, 18]. Theoretically, as long as circulating through the route of FUS beam, the disturbance for MBs induced by ultrasound energy will happen. It might lead to undesired cavitation effects at non-target area. But for phase shift droplets, the disturbance will occur only when the pressure is high enough to initiate ADV. This characteristic ensures the cavitation effects is limited in a stricter region and provides a promise for ultra-precise control of $\mathrm{BBB}$ opening. Moreover, the diameter of droplet can be easily manufactured into nanoscale size, so the residual nonexcited nanodroplets have the potential to extravasate into brain parenchyma through opened BBB.

The aim of this study was to synthesize one kind of polymeric phase shift perfluorocarbon nanodroplets and investigated whether it can serve as an alternative mediating agent for FUS induced BBB opening. Due to the applicable natural boiling point $\left(29^{\circ} \mathrm{C}\right)$, perfluoropentane (PFP) was chosen in this study. To achieve this aim, PEGylated PLGAbased PFP-encapsulated (PEG-PLGA-PFP) nanodroplets were prepared by a double emulsion method. Poly (ethylene glycol) - poly (lactide-co- glycolic acid) (PEG-PLGA), an amphiphilic copolymer which contained merits of PEG for long half-time and high stability in circulation $[19,20]$ and PLGA for good biocompatibility and biodegradability $[21,22]$, was selected as the shell materials.

\section{RESULTS}

\section{Characterization and thermal evaporation of PEG-PLGA-PFP nanodroplets}

The PEG-PLGA-PFP nanodroplets suspension (5 mg/ml) was shown ivory (Figure 1A). Transmission electron microscope (TEM) image (Figure 1B) revealed the spherical shell-core morphology of PEG-PLGA-PFP nanodroplet and the inner center dark mass of the sphere indicated the existence of liquid PFP core. The mean size distribution was $316.0 \pm 7.9 \mathrm{~nm}$ (Figure 1C) with a polydispersity index of , and the mean zeta potential was $-26.3 \pm 0.9 \mathrm{mV}$ (Figure 1D). The acoustic stability of nanodroplet suspension for 5-day storage at $4{ }^{\circ} \mathrm{C}$ had been checked (Supplementary Figure 1). The nanodroplets suspension $(1 \mathrm{mg} / \mathrm{ml})$ remained to be non-echo for two days after fabrication, but a very small amount of strong echoes came out three days after fabrication. As time went on, more and more strong echoes came out along with increasing of gray scale value. For preventing invalidation, therefore, PEG-PLGA-PFP nanodroplets should be used within two days after fabrication.

To further confirm the successful encapsulation of PFP and detected the liquid to gas phase shift temperature, PEG-PLGA-PFP nanodroplets were heated from room temperature $\left(20^{\circ} \mathrm{C}\right)$ to $55^{\circ} \mathrm{C}$. As shown in Figure $1 \mathrm{E}, 1 \mathrm{~F}$, no obvious bubbles were generated when the temperature reached $29^{\circ} \mathrm{C}$ and $37^{\circ} \mathrm{C}$. After the temperature reached $48^{\circ} \mathrm{C}$ (Figure $1 \mathrm{G}$ ), numerous of bubbles were generated which indicated the successful encapsulation of PFP. The paradoxical phenomenon that why the phase shift temperature was higher than the natural boiling point of PFP is attributed to the changes of interfacial surface tension [23, 24]. As the decrease of diameter into nano size, the boiling point of PFP was raised by the increasing partial pressure within the droplet. This characteristic also guaranteed the biostability of PEG-PLGA-PFP nanodroplets in circulation at physiological temperatures [25].

\section{FUS-induced phase shift in vitro}

Figure 2A illustrates the B-Mode and contrastenhanced ultrasound (CEUS) images of PEG-PLGA-PFP nanodroplets suspension $(1 \mathrm{mg} / \mathrm{ml})$ before and after FUS sonication at an acoustic pressure of $0.5 \mathrm{MPa}, 1.0 \mathrm{MPa}$ or $1.5 \mathrm{MPa}$ and sonication duration of $1 \mathrm{~min}, 3 \mathrm{~min}$ or $5 \mathrm{~min}$. Before FUS sonication, the B-Mode images showed PEG-PLGA-PFP nanodroplets were anechoic and no contrast-enhanced signals were detected. When acoustic pressure reached 1.0MPa, the B-mode and CEUS images showed noticeably increased echogenicity and contrast enhancement which indicated the successful phase shift and generation of microbubbles. The histograms showed the quantitative analysis of mean gray scale (Figure 2B) and mean acoustic intensity value (Figure 2C). Comparatively, the B-Mode and CEUS images were the strongest at acoustic pressure of 1.0MPa and sonication duration of $3 \mathrm{~min}$ (Table 1).

\section{Evan's blue extravasation}

The feasibility of in vivo BBB opening via FUS combined with PEG-PLGA-PFP nanodroplets was verified 
by qualitatively and quantitatively assessing extravasations of Evan's Blue (EB) in the sonicated side (right cerebral hemisphere) compared with the control (left cerebral hemisphere). The upper row of Figure $3 \mathrm{~A}$ showed the images of coronal brain sections and the EB extravasations indicated the area of BBB opening via FUS combined with nanodroplets. Figure 3B illustrates the quantitative analysis of the amount of EB extravasations in the sonicated side at an acoustic pressure of $0.5 \mathrm{MPa}, 1.0 \mathrm{MPa}$ or $1.5 \mathrm{MPa}$ with duration of $3 \mathrm{~min}$. At $0.5 \mathrm{MPa}$, the quantified $\mathrm{EB}$ extravasation showed no statistical significance compared with control and no brain tissues were stained by EB. When the acoustic pressure was increased to $1.0 \mathrm{MPa}$, the statistical significance was achieved $(P<0.05)$ and a very clear EB extravasation area within the right brain appeared. In the center of the extravasation area we could see a nearly $2 \mathrm{~mm}$ long but very narrow region which had a significant deep blue compared with the surrounding tissue. The long-narrow area was perpendicular to the FUS pathway and we believed this area demonstrated where BBB was successfully and intensively opened. As the acoustic pressure was increased to $1.5 \mathrm{MPa}$, the quantified $\mathrm{EB}$
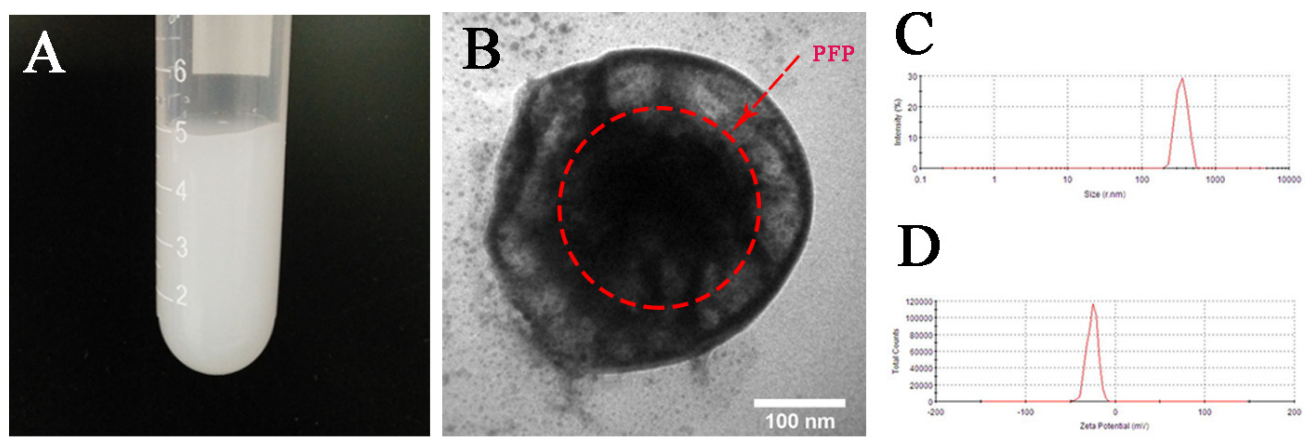

$\mathrm{D}$
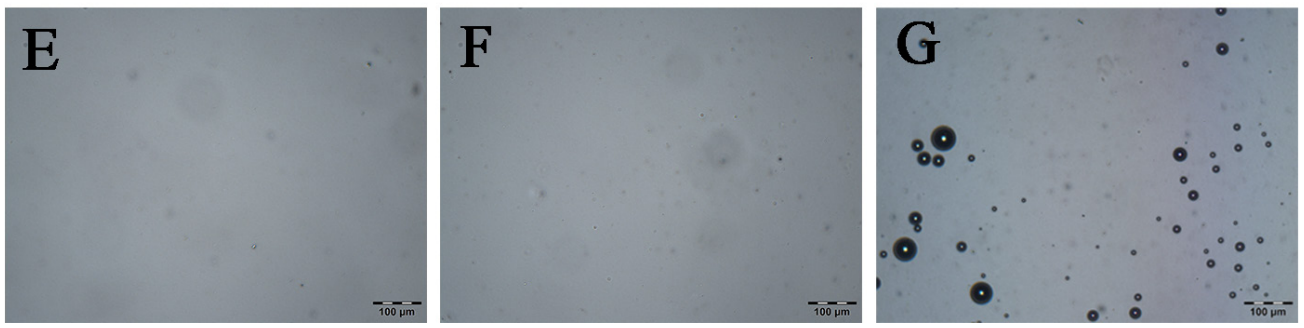

Figure 1: Characterization and in vitro thermal evaporation of PEG-PLGA-PFP nanodroplets. (A) A digital image of PEG-PLGA-PFP nanodroplets suspension $(5 \mathrm{mg} / \mathrm{ml}$ ). (B) A TEM image of PEG-PLGA-PFP nanodroplets. The red dotted circle shows the PFP inner core. (C and D) Size distribution and zeta potential of PEG-PLGA-PFP nanodroplets. (E-G) Microscope images $(\times 200)$ of PEGPLGA-PFP nanodroplets heated to $29^{\circ} \mathrm{C}(\mathrm{E}), 37^{\circ} \mathrm{C}(\mathrm{F})$ and $48^{\circ} \mathrm{C}(\mathrm{G})$, bar: $100 \mu \mathrm{m}$.

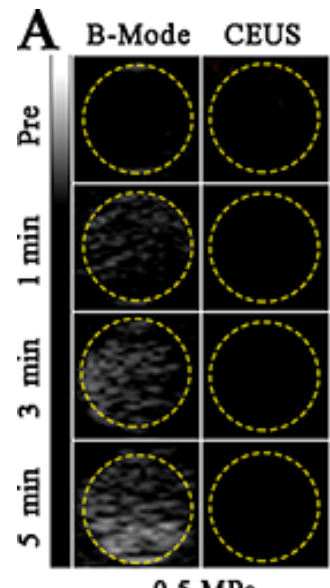

$0.5 \mathrm{MPa}$

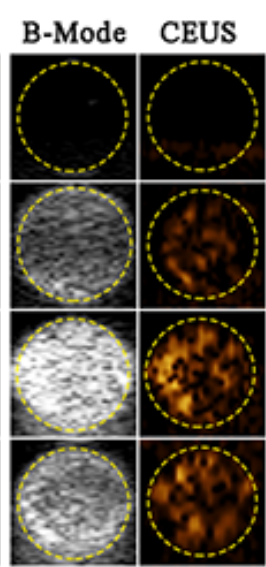

$1.0 \mathrm{MPa}$

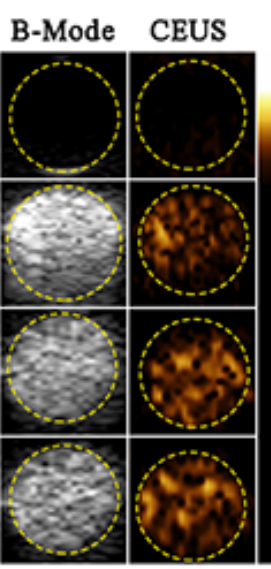

$1.5 \mathrm{MPa}$
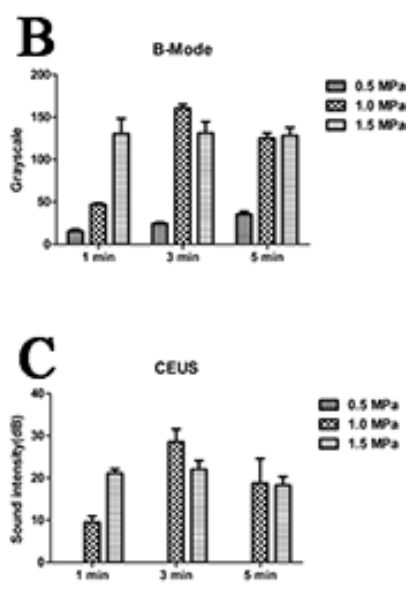

Figure 2: In vitro FUS-induced ADV of PEG-PLGA-PFP nanodroplets. (A) B-Mode and CEUS images of nanodroplets before and after FUS sonication. (B and C) The quantitative analysis of grayscales in B-Mode (B) and acoustic intensities in CEUS (C) of nanodroplets after FUS sonication. $(n=3)$. 
Table 1: Value of gray scale and acoustic intensity of PEG-PLGA-PFP nanodroplets suspension after FUS sonication

\begin{tabular}{cccc}
\hline Pressure (MPa) & Duration (min) & Gray scale & Acousitc intensity (dB) \\
\hline 0.5 & 1 & $15.4 \pm 2.4$ & 0 \\
0.5 & 3 & $24.4 \pm 1.5$ & 0 \\
0.5 & 5 & $35.6 \pm 3.2$ & 0 \\
1.0 & 1 & $47.0 \pm 1.6$ & $9.5 \pm 1.4$ \\
1.0 & 3 & $160.4 \pm 4.6$ & $28.6 \pm 3.0$ \\
1.0 & 5 & $125 \pm 6.1$ & $18.7 \pm 5.8$ \\
1.5 & 1 & $130.6 \pm 17.8$ & $21.1 \pm 1.0$ \\
1.5 & 3 & $131.0 \pm 13.5$ & $22.0 \pm 2.1$ \\
1.5 & 5 & $128.4 \pm 9.3$ & $18.3 \pm 2.0$ \\
\hline
\end{tabular}

$(n=3)$.

extravasations showed a significant increase and the central deep blue area apparently became longer and a little wider. Thus, 1.0MPa could be considered as the acoustic pressure threshold for FUS-induced BBB opening combined with PEG-PLGA-PFP nanodroplets in this study.

\section{Histological evaluation}

H\&E stain (Figure 3A, middle and bottom rows) was performed in order to assess the safety of this procedure. No erythrocyte extravasations or hemorrhages were observed at $0.5 \mathrm{MPa}$ and $1.0 \mathrm{MPa}$. When acoustic pressure was increased to $1.5 \mathrm{MPa}$, both the regions of BBB opening and the EB extravasation levels were significantly enhanced, but were accompanied by intracerebral hemorrhages and sporadic erythrocyte extravasations. These damages to the brain could be explained by the powerful inertial cavitation that disrupted the tight junctions too excessively in the focal region. Thus, 1.0 MPa may be a practicable acoustic pressure at

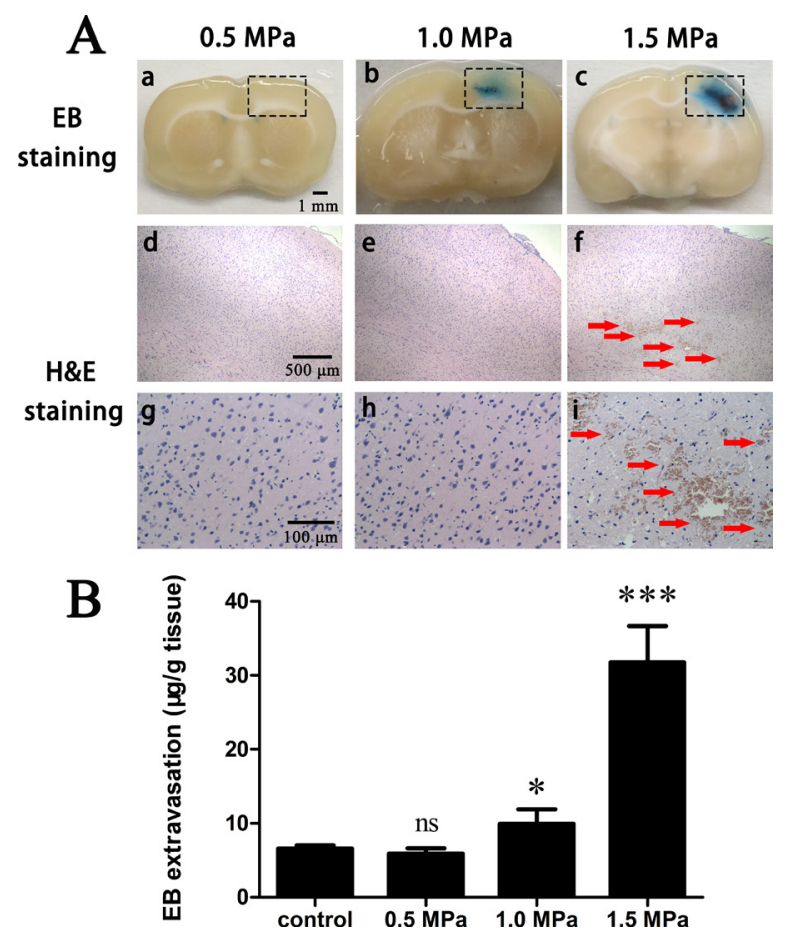

Figure 3: EB extravasation and H\&E stain. (A) FUS combined with PEG-PLGA-PFP nanodrplets induced BBB opening with acoustic pressure of $0.5 \mathrm{MPa}, 1.0 \mathrm{MPa}$ and 1.5 MPa. Significant EB extravasation was realized at acoustic pressure $1.0 \mathrm{MPa}(\mathrm{b})$ and increasing pressure would enhance the level (c). Intracerebral hemorrhages and sporadic erythrocyte extravasations (f, i) appeared at 1.5 MPa. Black rectangle: FUS sonication area. Upper row (a-c): gross brain section; middle row $(\mathrm{d}-\mathrm{g})$ ) enlarged $(\times 40)$ corresponding black rectangle region in H\&E stain; bottom row $(\mathrm{g}-\mathrm{i})$ : enlarged $(\times 200)$ corresponding black rectangle region in $\mathrm{H} \& \mathrm{E}$ stain. Red arrow: Intracerebral hemorrhages and sporadic erythrocyte extravasations. (B) Quantitative analysis of EB extravasation in vivo after FUS sonication combined with PEG-PLGA-PFP nanodroplets at an acoustic pressure of $0.5 \mathrm{MPa}, 1.0 \mathrm{MPa}$ or $1.5 \mathrm{MPa}$ with sonication duration of 3 min. (ns $=$ not significant, ${ }^{*} p<0.05,{ }^{* * *} p<0.001$ as compared with the control, $n=5$ ). 
which BBB could be successfully opening and biosecurity was satisfied at once, and we chose acoustic pressure of 1.0 $\mathrm{MPa}$ to investigate the time window.

\section{Time window}

Figure 4 demonstrates the amounts of EB extravasations changed as a function of time in the sonicated side at an acoustic pressure of $1.0 \mathrm{MPa}$ and sonication duration of 3 or $5 \mathrm{~min}$. EB extravasation reached the highest at beginning, and prolonged sonication duration could obviously enhance it. Then the values showed continuous declining which indicated the reestablishment of BBB integrity, especially in the first hour. At the time point of 1 hour, because no statistical difference was achieved as compared to the control, the reestablishment of BBB was seemed to be accomplished for brains underwent a 3 min sonication. For the other group, the time window was extended to 4 hours. Thus, increased sonication duration could enhance the level and prolong the time window of BBB opening.

\section{The difference of EB extravasation}

Figure 5 shows the difference of distribution pattern of EB extravasation induced by FUS combined with PEG-PLGA-PFP nanodroplets (Figure 5A) or lipidshelled MBs (Figure 5B). Both the deep blue regions were mostly distributed in the half-maximum of the pressure amplitude of the focal zone. For MBs, the long axis of the EB extravasation distribution followed the route of FUS beam, which was similar to our previous studies $[6,26]$. For PEG-PLGA-PFP nanodroplets, however, the long axis was perpendicular to the route, and the region of EB extravasation was firmly restricted in the central narrow area of the focal zone. Even when the acoustic power was increased to $1.5 \mathrm{MPa}$ at which severe brain injuries appeared, the minor axis of the region just became a little wider (Figure 4A).

\section{DISCUSSION}

FUS combined with lipid-shelled MBs has been verified as an effective and powerful tool for overcoming the barrier function of BBB at present. The main purpose of this study was to investigate whether PEGylated PLGA-based PFP-contained phase shift nanodroplets could be used to replace MBs as a novel mediating agent, and attempted to find out the difference of BBB opening regions between these two. In fact, this is not the first study that used phase shift nanodroplets as the mediating agent for FUS induced BBB opening. Chen et al. [27] had done an excellent job on it. They first generated one kind of lipid-shelled MBs in which contained perfluorobutane (PFB) gaseous cores, and then condensed the MBs into nanodroplets. However, due to the low boiling point $\left(-2^{\circ} \mathrm{C}\right)$ of PFB, they had to adopted relatively low temperature and high pressure for condensation. It raised demands on production conditions for other researchers. But the process provided by us could be performed just in an ice bath under normal atmospheric pressure. In the meantime, compared with lipid, the degradation process of PLGA can be regulated by adjusting the molecular weight or the ratio of lactide to glycolic acid (LA/GA). Increasing the molecular weight of PLGA will prolong the degradation time and raising the proportion of LA in PLGA will slow the degradation rate. These characteristics make PLGA an ideal skeleton material for drug delivery system [28].

The qualitative and quantitative analysis of EB extravasation confirmed the successful BBB opening

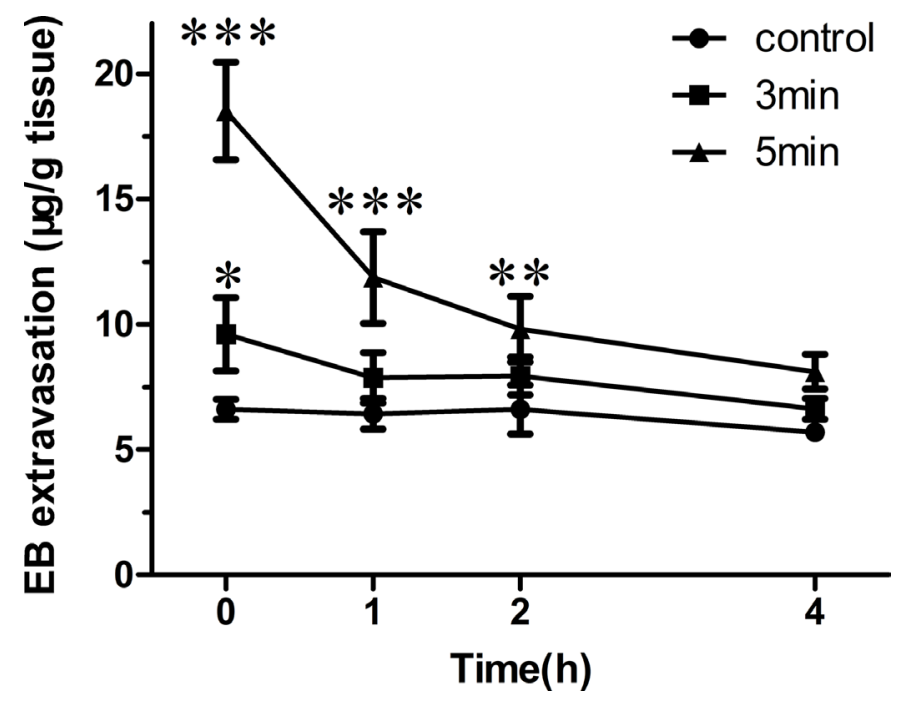

Figure 4: EB extravasation in the brain tissue was quantified $0,1,2$ or $4 \mathrm{~h}$ after 1.0 MPa sonication with duration of 3 or 5 min. ( ${ }^{*} p<0.05, * * p<0.01, * * * p<0.001$ as compared with the control, $n=3$ ). 
via FUS combined with PEG-PLGA-PFP nanodroplets when the acoustic pressure reached $1.0 \mathrm{MPa}$. It was bigger than the threshold $(0.45 \mathrm{MPa})$ that Chen et al. achieved. The main reason could be PFP has a higher boiling point $\left(29^{\circ} \mathrm{C}\right)$ so more pressure is needed. Secondly, the PLGA-based shell is more unyielding than lipid-based shell, so more power is required to break the shell down. Distributions of EB extravasation induced by nanodroplets were heavily limited in the central area of the focal zone, even when the pressure was increased to $1.5 \mathrm{MPa}$ at which intracerebral hemorrhages and erythrocyte extravasations appeared. For MBs, on the other hand, the EB extravasation appeared along the pathway of FUS in the focal zone. This difference between PEG-PLGA-PFP nanodroplets and MBs confirmed the theory that phase shift nanodroplets could only be excited when the acoustic pressure is high enough to excite it transforms from ground state (nanodroplet) to excited state (microbubble). This will be useful for high accuracy control of FUS induced $\mathrm{BBB}$ opening. Besides, the unscheduled second $\mathrm{BBB}$ opening area owing to the sound reflection on the skull base may be avoided. To our best knowledge, this is the second study to use phase shift nanodroplets as the mediating agents for FUS induced BBB opening and the first to use PLGA and PFP as essential ingredients. Interestingly and notablely, Airan et al. [29] had just described a novel technology that could silence seizures in an acute rat seizure model. They fabricated PFPencapsulated phase shift polymeric nanoparticle carriers of propofol. The carriers were excited by transcranial MRguided focused ultrasound (tcMRgFUS) sonication. And the propofol, which is a small enough to cross BBB, was targeted released during the liquid to gas phase transition. But the MRI data showed no evidence of BBB opening so that the brain homeostasis was maintained during the procedure. These two studies by Airan et al. and us are complementary, indicating that we might be about to reach the critical line. More studies on this are necessary.

Several major shortcomings of this study should be pointed out. Frist of all, a skull bone window was still a need. The energy attenuation induced by the presence of the skull seriously hindered the application of this technology. Second, lack of powerful guiding system made us can hardly focus on a uniform site of the brain. The tcMRgFUS has been proved to be an attractive and effective non-invasive technology for central nervous system diseases, such as seizure [29], trigeminal neuralgia [30], essential tremor [31], centrally located brain tumours [32], and Parkinson's disease [33, 34]. Thus, using tcMRgFUS may remedy these two defects at once. Shen et al. [35] demonstrated the successful delivery of liposomes with different sizes $(55 \mathrm{~nm}, 120 \mathrm{~nm}$ and $200 \mathrm{~nm}$ ) through opened BBB after FUS sonication $(1.282 \mathrm{MHz}+0.53$ or $0.64 \mathrm{MPa}+60 \mathrm{~s})$ in the presence of microbubbles. The nanodroplets we fabricated seemed to be too large. But as researches continue and technics advance, we believe that nanodroplets could one day successfully cross the opened BBB. The last and most serious shortcoming is the tremendously weak comparability of the compare, and the different materials used for fabrication makes it difficult to improve. The reason why we want to carry out this compare was due to the lack of studies on the difference of distribution pattern of BBB opening between nanoparticles and MBs. The results seemed to be positive for enhancing focusing performance by using phase shift nanodroplets. More high-quality comparisons are needed.

PLGA nanoparticles have been proved to be one kind of effective drug delivery system [36-39]. For the nanodroplets that we composed in this study, hydrophobic therapeutic agents can be easily encapsulated in the PLGA shell [40], and further surface modification with specific ligands would make these nanodroplets have active-targeting function [38]. Ho et al. [41] demonstrated that drug penetration can be enhanced via vascular disruption induced by using phase shift nanoscale droplets. Thus, drug loaded targeted phase shift nanodroplets combined with FUS for simultaneous BBB opening and extravascular therapy in the brain would be the next main direction for future study.

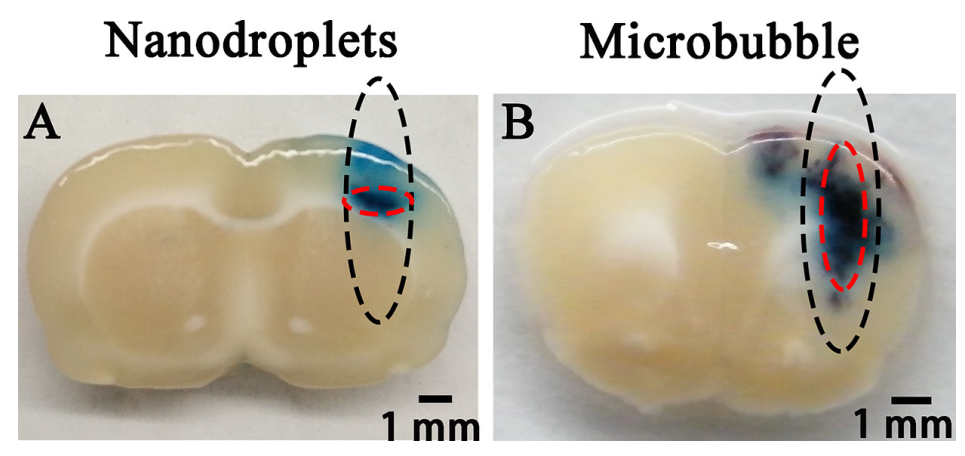

Figure 5: Distribution pattern of EB extravasation when PEG-PLGA-PFP nanodroplets (A) or lipid MBs (B) was used as mediating agent combined with FUS. The black dotted circles show the half-maximum of the pressure amplitude of the focal zone. The red dotted circles show the deep blue region of $\mathrm{EB}$ extravasation which indicates the location of $\mathrm{BBB}$ opening. 


\section{MATERIALS AND METHODS}

\section{Materials}

PEG-PLGA (5000/12000, lactide/glycolic acid = 50/50) copolymer was purchase from the Shan-dong Key Laboratory of Medical Polymer Materials (Shan-dong, China). PFP was purchased from Alfa Aesar (U.K.). Poly (vinyl alcohol) (PVA, 99\%, MW = 30,000-70,000 Da) and EB were obtained from Sigma-Aldrich Corp. (St. Louis, MO, USA). Self-made lipid-shelled MBs was provided by Guanjian Zhao. All chemicals used in this work were of analytical grade and were used as received.

\section{Preparation of PEG-PLGA-PFP nanodroplets}

Phase shift nanodroplets were prepared by a modified double emulsion/solvent evaporation method. Briefly, $50 \mathrm{mg}$ of PEG-PLGA was dissolved by $3 \mathrm{ml}$ dichloromethane (DCM) in a glass tube. The tube was inserted in the ice during dissolution. When PEG-PLGA was fully dissolved, $0.4 \mathrm{ml}$ of liquid PFP was added to the cold mixture and emulsified with an ultrasonic probe (SONICS \& MATERIALS, Inc., USA) (100 w, $5 \mathrm{~s}$ on $/ 5 \mathrm{~s}$ off, $1 \mathrm{~min}$ total). Once the sonication finished, $8 \mathrm{ml}$ of cold PVA (4\%) was added into the first emulsion immediately and carried out the second sonication $(100 \mathrm{w}, 5 \mathrm{~s}$ on $/ 5 \mathrm{~s}$ off, 2 min total). To evaporate DCM, the resulting solution was diluted with $20 \mathrm{ml}$ of cold PVA (4\%) and stirred gently for $6 \mathrm{~h}$. All of the processes mentioned above were carried out in ice bath. Subsequently, the final solution was centrifuged at $12,000 \mathrm{rpm}$ for $8 \mathrm{~min}$ at $4^{\circ} \mathrm{C}$. The supernatant was discarded and the precipitate was washed by deionized water. The process of centrifugation and washing was repeated three times. Finally, the nanodroplets were collected and stored at $4^{\circ} \mathrm{C}$.

\section{Characteristics of PEG-PLGA-PFP nanodroplets}

The size distribution and zeta potential of the nanodroplets were determined using a Malvern Zetasizer
Nano ZS unit (Malvern Instruments, UK). For each sample, the mean diameter of three determinations was calculated. Values reported were the mean \pm standard deviation of at least three different batches of nanodroplets. The morphology of PEG-PLGA-PFP nanodroplets was observed by TEM (Hitachi H-7600, Japan). To estimate the successful encapsulation of PFP, PEG-PLGA-PFP nanodroplets $(1 \mathrm{mg} / \mathrm{ml})$ were heated by a heating panel with a viewing hole and visualized under an inverted microscope.

\section{In vitro FUS-induced phase shift}

A portable FUS system was provided by the Department of Biomedical Engineering of Chongqing Medical University. Continuous FUS exposures were generated by a $1 \mathrm{MHz}$ single-element focused transducer (diameter $=40 \mathrm{~mm}$, focus length $=18 \mathrm{~mm}$ ). The diameter and length of the focal zone with half-maximum of the pressure amplitude were $2.2 \mathrm{~mm}$ and $6.9 \mathrm{~mm}$, respectively. The transducer was fixed in a handheld probe with a panhead and the center of the focal zone was about $8.2 \mathrm{~mm}$ away from the tip.

Figure 6A illustrated experimental setup for testing in vitro FUS-induced phase shift. Approximately $1 \mathrm{ml}(1 \mathrm{mg} / \mathrm{ml})$ of the PEG-PLGA-PFP nanodroplets suspension was injected into a hollow gel cuboid with strong sound transmission. Then the cuboid filled with nanodroplets underwent a FUS sonication along the long axis. The acoustic pressures ranged from $0.5 \mathrm{MPa}$ to $1.5 \mathrm{MPa}$ and the sonication time ranged from $1 \mathrm{~min}$ to $5 \mathrm{~min}$. Cross sectional B-Mode ultrasonography and contrast-enhanced ultrasonography (CEUS) were used to detect whether phase shift was realized after sonication. MyLab 90 (Esaote, Italy) with a linear probe (5-12 MHz) was used as the ultrasonic imaging system for observing and capturing, and experiments were repeated three times. The mean gray scale and sound intensity $(\mathrm{dB})$ of the nanodroplets suspension were then analyzed by the software DFY (invented by the Institution of Ultrasound Imaging of Chongqing Medical University, Chongqing, China) for further quantitatively analysis.
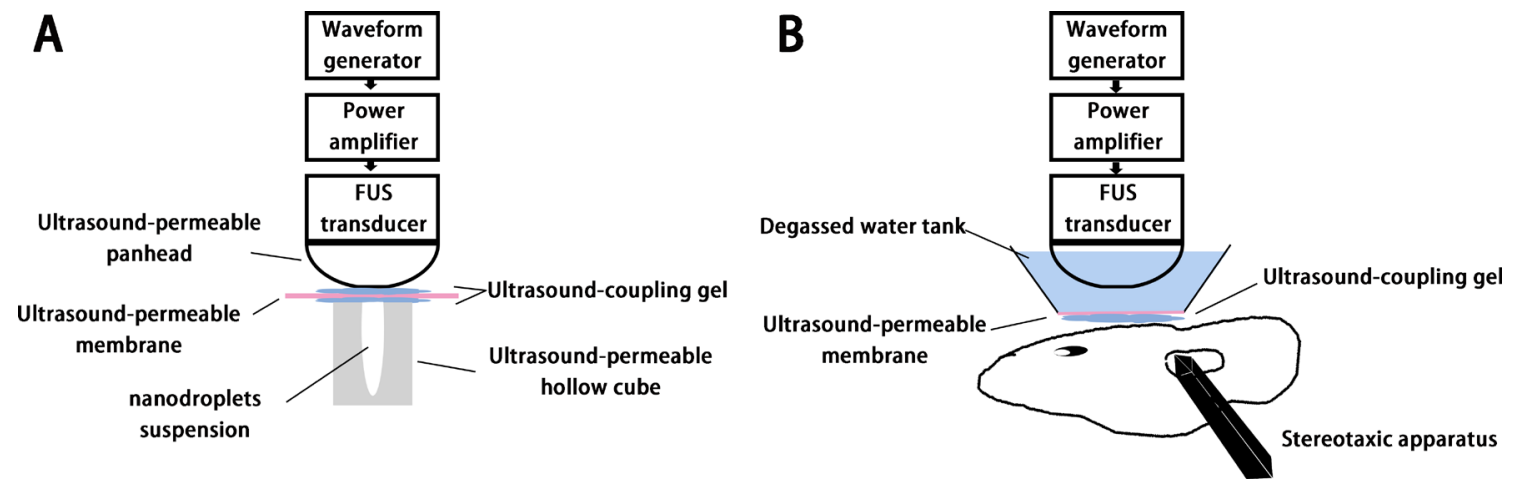

Figure 6: In vitro (A) and in vivo (B) experimental setup. 


\section{Animal preparation}

All procedures involving animals were approved by the Institutional Animal Care and Use Committee of Chongqing medical university. Male Sprague-Dawley (SD) rats weighing from $200 \mathrm{~g}$ to $250 \mathrm{~g}$ were used. The animals were housed in an IVC experimental animal house in which the temperature was maintained at $24^{\circ} \mathrm{C}$. Before sonication, each animal was anesthetized intraperitoneally with chloral hydrate $(400 \mathrm{mg} / \mathrm{kg})$ and then inserted a catheter (24G; Deltaflo, Italy) in the tail vein for injections. The fur on the scalp was removed with an electric clipper and depilatory cream. The head was fixed on a stereotaxic apparatus (ZS Dichuang, China) and the body temperature was maintained at $37^{\circ} \mathrm{C}$ using a heating pad. A window $(4 \mathrm{~mm} \times 5 \mathrm{~mm})$ at right parietal bone was opened for each animal and the right coronal suture crossed it along the middle line.

\section{In vivo experimental setup}

Figure 6B illustrated experimental setup for testing in vivo FUS-induced BBB opening. The same FUS system was used for in vivo protocols. For relatively precise targeting, its handheld probe was fixed on the left-right axis of the stereotaxic apparatus, and the targeted region was set at the position of $3.0 \mathrm{~mm}$ lateral and $0.5 \mathrm{~mm}$ anterior to the bregma, and $3.0 \mathrm{~mm}$ below the skull surface. The space between probe and skull surface was fill with a self-made degassed water tank whose bottom was an ultrasound permeable membrane, and ultrasound coupling gel was applied between the skull and the membrane.

\section{Confirmation of BBB opening and assessment of brain tissue injury}

Twenty-four rats were randomly divided into three groups. Continuous FUS sonication $(0.5,1.0$ or $1.5 \mathrm{MPa})$ was performed $20 \mathrm{~s}$ after intravenous administration of PEG-PLGA-PFP nanodroplets $(10 \mathrm{mg} / \mathrm{kg})$ into the tail vein, and the duration was fixed at $3 \mathrm{~min}$. To verify the successful opening of $\mathrm{BBB}$, rats were intravenously injected with EB $(100 \mathrm{mg} / \mathrm{kg})$ after FUS sonication immediately. Animals were sacrificed $4 \mathrm{~h}$ after the EB injection, and $0.9 \%$ normal saline was perfused into the left cardiac ventricle continuously until clear perfusion fluid flowed out of the right atrium. The brains were removed and sliced coronally after perfusion.

Five samples of each group were random selected, then divided into right and left hemispheres. The unsonicated left hemispheres were regarded as the control. Samples were weighed and then soaked in 50\% trichloroacetic acid solution. After homogenization and centrfugation, the extracted dye was diluted with ethanol (1:3), and the amount present was measured using a spectrophotometer at $620 \mathrm{~nm}$. The EB tissue content was quantifed via a linear regression standard curve derived from five concentrations of the dye and was denoted in terms of the amount per gram of tissue.

The rest three samples of each group were prepared for histological observation. Samples were fixed in $4 \%$ paraformaldehyde for $48 \mathrm{~h}$. After post fixation processing, the sections were embedded in paraffin, sectioned at $5 \mu \mathrm{m}$ thickness, and stained with hematoxylin and eosin (H\&E). Histologic evaluation was performed by an author who was blind to the parameters of sonication.

\section{Time window}

Twenty-four rats were were randomly divided into two groups. Continuous FUS sonication with duration of 3 or $5 \mathrm{~min}$ at $1.0 \mathrm{MPa}$ was performed $20 \mathrm{~s}$ after intravenous administration of PEG-PLGA-PFP nanodroplets $(10 \mathrm{mg} / \mathrm{kg})$ into the tail vein. At 4 time points $(0,1,2$ or $4 \mathrm{~h}$ ) after sonication, three rats of each group were selected for procedure of EB extravasation evaluation.

\section{Distribution pattern of EB extravasation}

For investigating the difference of distribution pattern of EB extravasation, we used another three SD rats underwent a continuous FUS sonication combined with conventional self-made lipid MBs. The MBs were provided by Guanjian Zhao in our group. The concentration of MBs was adjusted to $10^{8} / \mathrm{ml}$ and the dose used for each rat was set at $2 \times 10^{8} / \mathrm{kg}$. The acoustic pressure was set at $1.0 \mathrm{MPa}$, and the sonication duration was set at $40 \mathrm{sec}$ according to our previous study $[6,26]$.

\section{Statistical analysis}

An unpaired student's $t$ test was used for Statistical analysis and a probability (P) less than 0.05 was considered significant. Results were expressed as mean standard \pm deviation (SD).

\section{CONCLUSIONS}

In this study, PEGylated PLGA-based PFPencapsulated phase shift nanodroplets were successfully fabricated. Significant EB extravasation and satisfied biosecurity could be achieved by the systematic administration of this nanodroplets combined with FUS at acoustic pressure of $1.0 \mathrm{MPa}$. Prolonged duration of sonication could broaden the time window of BBB opening. In summary, these promising results indicate that the PEG-PLGA-PFP nanodroplets could serve as an effective alternative mediating agent and have great potential for ultra-precise control of BBB opening and target drug delivery. 


\section{ACKNOWLEDGMENTS AND FUNDING}

This work was supported by Grants from the National Natural Science Foundation of China (Nos. 81201066, 81371674, 81471676, and 81401505) and the Chongqing Natural Science Foundation Project (Nos. CSTC2013jcyjA10054 and CSTC2014jcyjA10050).

\section{CONFLICTS OF INTEREST}

We declare that we have no financial and personal relationships with other people or organizations that can inappropriately influence our work, there is no professional or other personal interest of any nature or kind in any product, service and/or company that could be construed as influencing the position presented in, or the review of, the manuscript entitled, "PEGylated PLGAbased phase shift nanodroplets combined with focused ultrasound for blood brain barrier opening in rats".

\section{REFERENCES}

1. Fan CH, Cheng YH, Ting CY, Ho YJ, Hsu PH, Liu HL, Yeh CK. Ultrasound/Magnetic Targeting with SPIO-DOXMicrobubble Complex for Image-Guided Drug Delivery in Brain Tumors. Theranostics. 2016; 6:1542-56.

2. Fan $\mathrm{CH}$, Ting $\mathrm{CY}$, Lin HJ, Wang CH, Liu HL, Yen TC, Yeh CK. SPIO-conjugated, doxorubicin-loaded microbubbles for concurrent MRI and focused-ultrasound enhanced brain-tumor drug delivery. Biomaterials. 2013; 34:3706-15.

3. Liu HL, Fan $\mathrm{CH}$, Ting $\mathrm{CY}$, Yeh CK. Combining microbubbles and ultrasound for drug delivery to brain tumors: current progress and overview. Theranostics. 2014; 4:432-44.

4. Jordao JF, Thevenot E, Markham-Coultes K, Scarcelli T, Weng YQ, Xhima K, O'Reilly M, Huang Y, McLaurin J, Hynynen K, Aubert I. Amyloid-beta plaque reduction, endogenous antibody delivery and glial activation by braintargeted, transcranial focused ultrasound. Exp Neurol. 2013; 248:16-29.

5. Jordao JF, Ayala-Grosso CA, Markham K, Huang Y, Chopra R, McLaurin J, Hynynen K, Aubert I. Antibodies targeted to the brain with image-guided focused ultrasound reduces amyloid-beta plaque load in the TgCRND8 mouse model of Alzheimer's disease. PLoS One. 2010; 5:e10549.

6. Huang Q, Deng J, Wang F, Chen S, Liu Y, Wang Z, Wang Z, Cheng Y. Targeted gene delivery to the mouse brain by MRI-guided focused ultrasound-induced blood-brain barrier disruption. Exp Neurol. 2012; 233:350-6.

7. Fan CH, Chang EL, Ting CY, Lin YC, Liao EC, Huang CY, Chang YC, Chan HL, Wei KC, Yeh CK. Folate-conjugated gene-carrying microbubbles with focused ultrasound for concurrent blood-brain barrier opening and local gene delivery. Biomaterials. 2016; 106:46-57.
8. Burgess A, Ayala-Grosso CA, Ganguly M, Jordao JF, Aubert I, Hynynen K. Targeted delivery of neural stem cells to the brain using MRI-guided focused ultrasound to disrupt the blood-brain barrier. PLoS One. 2011; 6:e27877.

9. Sboros V. Response of contrast agents to ultrasound. Adv Drug Deliv Rev. 2008; 60:1117-36.

10. Husseini GA, Diaz de la Rosa MA, Richardson ES, Christensen DA, Pitt WG. The role of cavitation in acoustically activated drug delivery. J Control Release. 2005; 107:253-61.

11. Sirsi S, Feshitan J, Kwan J, Homma S, Borden M. Effect of microbubble size on fundamental mode high frequency ultrasound imaging in mice. Ultrasound Med Biol. 2010; 36:935-48

12. Seda R, Li DS, Fowlkes JB, Bull JL. Characterization of Bioeffects on Endothelial Cells under Acoustic Droplet Vaporization. Ultrasound Med Biol. 2015.

13. Kripfgans OD, Fowlkes JB, Miller DL, Eldevik OP, Carson PL. Acoustic droplet vaporization for therapeutic and diagnostic applications. Ultrasound Med Biol. 2000; 26:1177-89.

14. Giesecke T, Hynynen K. Ultrasound-mediated cavitation thresholds of liquid perfluorocarbon droplets in vitro. Ultrasound Med Biol. 2003; 29:1359-65.

15. Fabiilli ML, Haworth KJ, Fakhri NH, Kripfgans OD, Carson PL, Fowlkes JB. The role of inertial cavitation in acoustic droplet vaporization. IEEE Trans Ultrason Ferroelectr Freq Control. 2009; 56:1006-17.

16. Kripfgans OD, Fabiilli ML, Carson PL, Fowlkes JB. On the acoustic vaporization of micrometer-sized droplets. J Acoust Soc Am. 2004; 116:272-81.

17. Shpak O, Verweij M, Vos HJ, de Jong N, Lohse D, Versluis M. Acoustic droplet vaporization is initiated by superharmonic focusing. Proc Natl Acad Sci U S A. 2014; 111:1697-702.

18. Lin CY, Pitt WG. Acoustic droplet vaporization in biology and medicine. Biomed Res Int. 2013; 2013:404361.

19. Gref R, Luck M, Quellec P, Marchand M, Dellacherie E, Harnisch S, Blunk T, Muller RH. 'Stealth' corona-core nanoparticles surface modified by polyethylene glycol (PEG): influences of the corona (PEG chain length and surface density) and of the core composition on phagocytic uptake and plasma protein adsorption. Colloids Surf B Biointerfaces. 2000; 18:301-13.

20. Mahapatro A, Singh DK. Biodegradable nanoparticles are excellent vehicle for site directed in-vivo delivery of drugs and vaccines. J Nanobiotechnology. 2011; 9:55.

21. Danhier F, Lecouturier N, Vroman B, Jerome C, MarchandBrynaert J, Feron O, Preat V. Paclitaxel-loaded PEGylated PLGA-based nanoparticles: in vitro and in vivo evaluation. J Control Release. 2009; 133:11-7.

22. Fonseca C, Simoes S, Gaspar R. Paclitaxel-loaded PLGA nanoparticles: preparation, physicochemical characterization and in vitro anti-tumoral activity. J Control Release. 2002; 83:273-86. 
23. Rapoport N. Drug-Loaded Perfluorocarbon Nanodroplets for Ultrasound-Mediated Drug Delivery. Adv Exp Med Biol. 2016; 880:221-41.

24. Zhou Y. Application of acoustic droplet vaporization in ultrasound therapy. J Ther Ultrasound. 2015; 3:20.

25. Zhang N, Cai X, Gao W, Wang R, Xu C, Yao Y, Hao L, Sheng D, Chen H, Wang Z, Zheng Y. A Multifunctional Theranostic Nanoagent for Dual-Mode Image-Guided HIFU/Chemo-Synergistic Cancer Therapy. Theranostics. 2016; 6:404-17.

26. Deng J, Huang Q, Wang F, Liu Y, Wang Z, Wang Z, Zhang Q, Lei B, Cheng Y. The role of caveolin-1 in bloodbrain barrier disruption induced by focused ultrasound combined with microbubbles. J Mol Neurosci. 2012; 46:677-87.

27. Chen CC, Sheeran PS, Wu SY, Olumolade OO, Dayton PA, Konofagou EE. Targeted drug delivery with focused ultrasound-induced blood-brain barrier opening using acoustically-activated nanodroplets. J Control Release. 2013; 172:795-804.

28. Shive MS, Anderson JM. Biodegradation and biocompatibility of PLA and PLGA microspheres. Adv Drug Deliv Rev. 1997; 28:5-24.

29. Airan RD, Meyer RA, Ellens NP, Rhodes KR, Farahani K, Pomper MG, Kadam SD, Green JJ. Noninvasive Targeted Transcranial Neuromodulation via Focused Ultrasound Gated Drug Release from Nanoemulsions. Nano Lett. 2017; 17:652-9.

30. Monteith SJ, Medel R, Kassell NF, Wintermark M, Eames M, Snell J, Zadicario E, Grinfeld J, Sheehan JP, Elias WJ. Transcranial magnetic resonance-guided focused ultrasound surgery for trigeminal neuralgia: a cadaveric and laboratory feasibility study. J Neurosurg. 2013; 118:319-28.

31. Gallay MN, Moser D, Rossi F, Pourtehrani P, Magara AE, Kowalski M, Arnold A, Jeanmonod D. Incisionless transcranial MR-guided focused ultrasound in essential tremor: cerebellothalamic tractotomy. J Ther Ultrasound. 2016; 4:5.

32. Coluccia D, Fandino J, Schwyzer L, O'Gorman R, Remonda L, Anon J, Martin E, Werner B. First noninvasive thermal ablation of a brain tumor with MR-guided focused ultrasound. J Ther Ultrasound. 2014; 2:17.
33. Kyriakou A, Neufeld E, Werner B, Paulides MM, Szekely G, Kuster N. A review of numerical and experimental compensation techniques for skull-induced phase aberrations in transcranial focused ultrasound. Int $\mathbf{J}$ Hyperthermia. 2014; 30:36-46.

34. Moser D, Zadicario E, Schiff G, Jeanmonod D. MR-guided focused ultrasound technique in functional neurosurgery: targeting accuracy. J Ther Ultrasound. 2013; 1:3.

35. Shen Y, Guo J, Chen G, Chin CT, Chen X, Chen J, Wang F, Chen S, Dan G. Delivery of Liposomes with Different Sizes to Mice Brain after Sonication by Focused Ultrasound in the Presence of Microbubbles. Ultrasound Med Biol. 2016; 42:1499-511.

36. Chen Z, Patel JM, Noble PW, Garcia C, Hong Z, Hansen JE, Zhou J. A lupus anti-DNA autoantibody mediates autocatalytic, targeted delivery of nanoparticles to tumors. Oncotarget. 2016; 7:59965-75. doi: 10.18632/oncotarget.11015.

37. Aravind A, Varghese SH, Veeranarayanan S, Mathew A, Nagaoka Y, Iwai S, Fukuda T, Hasumura T, Yoshida Y, Maekawa T, Kumar DS. Aptamer-labeled PLGA nanoparticles for targeting cancer cells. Cancer Nanotechnol. 2012; 3:1-12.

38. Guo J, Gao X, Su L, Xia H, Gu G, Pang Z, Jiang X, Yao L, Chen J, Chen H. Aptamer-functionalized PEG-PLGA nanoparticles for enhanced anti-glioma drug delivery. Biomaterials. 2011; 32:8010-20.

39. He Z, Huang J, Xu Y, Zhang X, Teng Y, Huang C, Wu Y, Zhang X, Zhang H, Sun W. Co-delivery of cisplatin and paclitaxel by folic acid conjugated amphiphilic PEG-PLGA copolymer nanoparticles for the treatment of non-small lung cancer. Oncotarget. 2015; 6:42150-68. doi: 10.18632/ oncotarget.6243.

40. Xu Y, Shen M, Li Y, Sun Y, Teng Y, Wang Y, Duan Y. The synergic antitumor effects of paclitaxel and temozolomide co-loaded in mPEG-PLGA nanoparticles on glioblastoma cells. Oncotarget. 2016; 7:20890-901.

41. Ho YJ, Chang YC, Yeh CK. Improving Nanoparticle Penetration in Tumors by Vascular Disruption with Acoustic Droplet Vaporization. Theranostics. 2016; 6:392-403. doi: 10.18632/oncotarget.7896. 